\title{
Solving Variational Inclusions by a Method Obtained Using a Multipoint Iteration Formula
}

\author{
Catherine CABUZEL and Alain PIÉTRUS \\ Laboratoire Analyse, Optimisation, Contrôle \\ Université des Antilles et de la Guyane \\ Département de Mathématiques et Informatique \\ Campus de Fouillole \\ F-97159 Pointe-à-Pitre - France \\ catherine.zebre@univ-ag.fr apietrus@univ-ag.fr
}

Received: March 26, 2007

Accepted: February 18, 2008

\section{ABSTRACT}

This paper deals with variational inclusions of the form: $0 \in f(x)+F(x)$ where $f$ is a single function admitting a second order Fréchet derivative and $F$ is a set-valued map acting in Banach spaces. We prove the existence of a sequence $\left(x_{k}\right)$ satisfying $0 \in f\left(x_{k}\right)+\sum_{i=1}^{M} a_{i} \nabla f\left(x_{k}+\beta_{i}\left(x_{k+1}-x_{k}\right)\right)\left(x_{k+1}-x_{k}\right)+F\left(x_{k+1}\right)$ where the single-valued function involved in this relation is an approximation of the function $f$ based on a multipoint iteration formula and we show that this method is locally cubically convergent.

Key words: set-valued mapping, generalized equations, pseudo-Lipschitz maps, multipoint iteration formula.

2000 Mathematics Subject Classification: 49J53, 47H04, 65K10.

\section{Introduction}

This paper deals with the problem of approximating a solution of the "abstract" generalized equation

$$
0 \in f(x)+F(x),
$$

where $f$ is a function from $X$ into $Y$, which admits a second order Fréchet derivative, $F$ is a set-valued map from $X$ to the subsets of $Y$ with closed graph, and $X, Y$ are two Banach spaces.

Let us recall that equation (1) is an abstract model for various problems. 
- When $F=\{0\}$, (1) is an equation,

- when $F$ is the positive orthant in $\mathbb{R}^{m},(1)$ is a system of inequalities,

- when $F$ is the normal cone to a convex and closed set in $X,(1)$ may represent variational inequalities.

When the Fréchet derivative $\nabla f$ of $f$ is locally Lipschitz, Dontchev [3,4] associates to (1) a Newton-type method based on a partial linearization which provides a local quadratic convergence. Following his work, Piétrus [16] obtains a Newton-type sequence which converges whenever $\nabla f$ satisfies a Hölder-type condition and in [15] he proves the stability.

Using a second-degree Taylor polynomial expansion of $f$ at $x_{k}$, Geoffroy, Hilout, and Piétrus [7] introduced a method involving the second order Fréchet derivative and, when $\nabla^{2} f$ is lipschitz, they obtained a cubic convergence. In [8] they proved the stability of the method and in [10] they showed that the previous method is superquadratic when $\nabla^{2} f$ satisfies an Hölder condition. Lately, Jean-Alexis presented in [12] a method without second order derivative, which is also cubically convergent and Geoffroy, Jean-Alexis and Piétrus showed the stability of this method in [9]. Our method generalizes this idea by taking more iterates.

For solving (1), we fix an integer $M>1$ and we consider the sequence

$$
0 \in f\left(x_{k}\right)+\sum_{i=1}^{M} a_{i} \nabla f\left(x_{k}+\beta_{i}\left(x_{k+1}-x_{k}\right)\right)\left(x_{k+1}-x_{k}\right)+F\left(x_{k+1}\right)
$$

where $\left(a_{i}\right)_{1 \leq i \leq M}$ and $\left(\beta_{i}\right)_{1 \leq i \leq M}$ are two sequences of real numbers satisfying

$$
\sum_{i=1}^{M} a_{i}=1 \quad \text { and } \quad \sum_{i=1}^{M} a_{i} \beta_{i}=\frac{1}{2}
$$

The inspiration for considering such a method comes from a multipoint iteration formula given in [19] for approximating $f$.

Let us remark that there is no second order Fréchet derivative of $f$ in this approximation of $f$ but only $M$ values of $\nabla f$ computed at $\left(x_{k}+\beta_{i}\left(x_{k+1}-x_{k}\right)\right), 1 \leq i \leq M$.

The paper is organized as follows: in section 2 , we give some definitions and recall a few preliminary results as a fixed-point theorem (lemma 1.3) which is the main tool for proving the existence and the convergence of the sequence defined by (2) to a solution of the equation (1); we also make some fundamental assumptions on $f$.

The existence and the convergence of the previous sequence defined by (2) are developed in section 3. Moreover, we prove that the convergence of this method is of order 3 .

Let us recall some notation: 
- The distance from a point $x$ to a set $A$ in the metric space $(Z, \rho)$ is defined by

$$
\operatorname{dist}(x, A)=\inf \{\rho(x, y), y \in A\} \text {; }
$$

- The excess $e$ from the set $A$ to the set $C$ is given by

$$
e(A, C)=\sup \{\operatorname{dist}(x, A), x \in C\} ;
$$

- Let $\Lambda: X \rightrightarrows Y$ be a set-valued map, we write

$$
\operatorname{graph} \Lambda=\{(x, y) \in X \times Y, y \in \Lambda(x)\} \quad \text { and } \quad \Lambda^{-1}(y)=\{x \in X, y \in \Lambda(x)\} ;
$$

- $B_{r}(x)$ is the closed ball centered at $x$ with radius $r$;

- The norms in the Banach spaces $X$ and $Y$ are both denoted by $\|\cdot\|$.

\section{Definitions and preliminary results}

In this section, we collect some results that we will need to prove our main theorem.

Definition 1.1. A set-valued $\Lambda$ is pseudo-Lipschitz around $\left(x_{0}, y_{0}\right) \in$ graph $\Lambda$ with modulus $L$ if there exist constants $a$ and $b$ such that

$$
\sup _{y \in \Lambda\left(x^{\prime}\right) \cap B_{a}\left(y_{0}\right)} \operatorname{dist}\left(y, \Lambda\left(x^{\prime \prime}\right)\right) \leq L\left\|x^{\prime}-x^{\prime \prime}\right\|, \quad \text { for all } x^{\prime} \text { and } x^{\prime \prime} \text { in } B_{b}\left(x_{0}\right) .
$$

Using the excess, we have an equivalent definition replacing the inequality (4) by

$$
e\left(\Lambda\left(x^{\prime}\right) \cap B_{a}\left(y_{0}\right), \Lambda\left(x^{\prime \prime}\right)\right) \leq L\left\|x^{\prime}-x^{\prime \prime}\right\|, \quad \text { for all } x^{\prime} \text { and } x^{\prime \prime} \text { in } B_{b}\left(x_{0}\right) .
$$

The pseudo-Lipschitz property has been introduced J.-P. Aubin and he was the first to define this concept as a continuity property. Let us note that sometimes this property is also called "Aubin continuity." Characterizations of the pseudo-Lipschitz property are also obtained by Rockafellar in $[17,18]$ using the Lipschitz continuity of the distance function dist $(y, \Lambda(x))$ around $\left(x_{0}, y_{0}\right)$ and by Mordukhovich in $[13,14]$ using the concept of coderivative of multifunctions. The Aubin-continuity of $F$ is equivalent to the metric regularity of $F^{-1}$. Lately, Dontchev, Quincampoix, and Zlateva gave in [6] a derivative criterion for the metric regularity of set-valued mappings based on works of Aubin and co-authors. Fore more details and applications of this property, the reader could also refer to $[1,2,5]$.

Definition 1.2. We say that a function $f$ from a metric space $(X, \rho)$ into a metric space $(Y, d)$ is strictly stationary at $x_{0} \in X$ if, for every $\varepsilon>0$, there exists $\delta>0$ such that

$$
d\left(f\left(x_{1}\right), f\left(x_{2}\right)\right) \leq \epsilon \rho\left(x_{1}, x_{2}\right) \quad \text { whenever } \quad \rho\left(x_{i}, x_{0}\right)<\delta, \quad i=1,2 .
$$


Lemma 1.3. Let $(Z, \rho)$ be a complete metric space, let $\phi$ be a set-valued map from $Z$ into the closed subsets of $Z$, let $\eta_{0} \in Z$ and let $r$ and $\lambda$ be such that $0 \leq \lambda<1$ and

(i) $\operatorname{dist}\left(\eta_{0}, \phi\left(\eta_{0}\right)\right) \leq r(1-\lambda)$,

(ii) $e\left(\phi\left(x_{1}\right) \cap B_{r}\left(\eta_{0}\right), \phi\left(x_{2}\right)\right) \leq \lambda \rho\left(x_{1}, x_{2}\right), \forall x_{1}, x_{2} \in B_{r}\left(\eta_{0}\right)$,

then $\phi$ has a fixed-point in $B_{r}\left(\eta_{0}\right)$. That is, there exists $x \in B_{r}\left(\eta_{0}\right)$ such that $x \in \phi(x)$. If $\phi$ is single-valued, then $x$ is the unique fixed point of $\phi$ in $B_{r}\left(\eta_{0}\right)$.

The proof of lemma 1.3 is given in [5] employing the standard iterative concept for nonexpansive mappings. This fixed-point lemma is a generalization of the fixedpoint theorem in Ioffe-Tikhomirov [11] where in (ii) the excess $e$ is replaced by the Haussdorf distance.

We make the following assumptions on a neighborhood $\Omega$ of $x^{*}$ :

$(\mathcal{H} 1) \nabla^{2} f$ is $K_{2}$-Lipschtiz in $\Omega$

$(\mathcal{H} 2) \quad F$ is a set-valued map with closed graph.

$(\mathcal{H} 3)(f+F)^{-1}$ is pseudo-Lipschitz around $\left(0, x^{*}\right)$ with constants $a, b$ and modulus $L$.

We also define the following functions:

$$
\begin{aligned}
\Lambda_{k}(x) & =f\left(x_{k}\right)+\sum_{i=1}^{M} a_{i} \nabla f\left(x_{k}+\beta_{i}\left(x-x_{k}\right)\right)\left(x-x_{k}\right) \\
\Lambda_{x^{*}}(x) & =f\left(x^{*}\right)+\sum_{i=1}^{M} a_{i} \nabla f\left(x^{*}+\beta_{i}\left(x-x^{*}\right)\right)\left(x-x^{*}\right) \\
Q(x) & =\Lambda_{x^{*}}(x)+F(x)
\end{aligned}
$$

and

$$
\Psi_{k}(x)=Q^{-1}\left(\Lambda_{x^{*}}(x)-\Lambda_{k}(x)\right)
$$

\section{Description of the method and convergence results}

- From a starting point $x_{0}$ in a neighborhood of a solution $x^{*}$ of (1), applying lemma 1.3, we show that $\Psi_{0}$ possesses a fixed point $x_{1}$.

- From a current iterate $x_{k}$ generated by (2) and a function $\Psi_{k}$ defined on $X$ by (5), applying lemma 1.3, we obtain the existence of the next iterate $x_{k+1}$ which is a fixed point of $\Psi_{k}$.

The main result of this study reads as follows: 
Theorem 2.1. Let $x^{*}$ be a solution of $(1)$, and suppose that $(\mathcal{H} 1)-(\mathcal{H} 3)$ are satisfied. Then for all $c>\frac{L K_{2}}{6}\left(1+3 \sum_{i=1}^{M}\left|a_{i}\right| \beta_{i}^{2}\right)$, one can find $\delta>0$ such that for every starting point $x_{0} \in B_{\delta}\left(x^{*}\right)$, there exists a sequence $\left(x_{k}\right)_{k \geq 0}$ defined by (2) which satisfies

$$
\left\|x_{k+1}-x^{*}\right\| \leq c\left\|x_{k}-x^{*}\right\|^{3} .
$$

Before proving the theorem, we show that

Proposition 2.2. The following are equivalent:

(i) $\left[f\left(x^{*}\right)+\sum_{i=1}^{M} a_{i} \nabla f\left(x^{*}+\beta_{i}\left(\cdot-x^{*}\right)\right)\left(\cdot-x^{*}\right)+F(\cdot)\right]^{-1}$ is pseudo-Lipschitz around $\left(y^{*}, x^{*}\right)$,

(ii) $(f+F)^{-1}$ is pseudo-Lipschitz around $\left(y^{*}, x^{*}\right)$.

Proof. According to [5, corollary 2], it suffices to prove, under the assumptions of theorem 2.1, that the function $h$ defined by

$$
h(x)=f(x)-\Lambda_{x^{*}}(x)
$$

is strictly stationary at $x^{*}$. Without lose of generality, we can suppose that the neighborhood $\Omega$ is bounded. This implies, thanks to $(\mathcal{H} 1)$, that $\nabla^{2} f$ is bounded on $\Omega$ by $K_{1}$ which implies that $\nabla f$ is $K_{1}$-Lipschitz on $\Omega$.

Let $\epsilon>0$ and let us set

$$
\alpha_{1}=\frac{\epsilon}{2 K_{1}\left[\sum_{i=1}^{M}\left|a_{i}\right|\left(1+\left|\beta_{i}\right|\right)\right]} .
$$

Fix $\delta>0$ such that $\delta \leq \min \left\{a ; \alpha_{1}\right\}$ where $a$ is given by the assumption $(\mathcal{H} 3)$ and let $x_{1}, x_{2} \in B_{\delta}\left(x^{*}\right)$.

$$
\begin{aligned}
\left\|h\left(x_{1}\right)-h\left(x_{2}\right)\right\|= & \left\|f\left(x_{1}\right)-\Lambda_{x^{*}}\left(x_{1}\right)-f\left(x_{2}\right)+\Lambda_{x^{*}}\left(x_{2}\right)\right\| \\
\leq & \left\|f\left(x_{1}\right)-f\left(x_{2}\right)-\sum_{i=1}^{M} a_{i} \nabla f\left(x^{*}+\beta_{i}\left(x_{1}-x^{*}\right)\right)\left(x_{1}-x_{2}\right)\right\| \\
& +\|\left(\sum _ { i = 1 } ^ { M } a _ { i } \left(\nabla f\left(x^{*}+\beta_{i}\left(x_{2}-x^{*}\right)\right)\right.\right. \\
& \left.-\nabla f\left(x^{*}+\beta_{i}\left(x_{1}-x^{*}\right)\right)\right)\left(x_{2}-x^{*}\right) \| .
\end{aligned}
$$

Let us set

$$
\begin{aligned}
& A=\left\|f\left(x_{1}\right)-f\left(x_{2}\right)-\sum_{i=1}^{M} a_{i} \nabla f\left(x^{*}+\beta_{i}\left(x_{1}-x^{*}\right)\right)\left(x_{1}-x_{2}\right)\right\|, \\
& B=\left\|\left(\sum_{i=1}^{M} a_{i} \nabla f\left(x^{*}+\beta_{i}\left(x_{2}-x^{*}\right)\right)-\sum_{i=1}^{M} a_{i} \nabla f\left(x^{*}+\beta_{i}\left(x_{1}-x^{*}\right)\right)\right)\left(x_{2}-x^{*}\right)\right\| .
\end{aligned}
$$


Using (3), we obtain

$$
A=\left\|\sum_{i=1}^{M} a_{i}\left(f\left(x_{1}\right)-f\left(x_{2}\right)-\nabla f\left(x^{*}+\beta_{i}\left(x_{1}-x^{*}\right)\right)\left(x_{1}-x_{2}\right)\right)\right\|,
$$

then,

$$
A \leq \sum_{i=1}^{M}\left|a_{i}\right|\left\|x_{1}-x_{2}\right\| \int_{0}^{1}\left\|\nabla f\left(x_{2}+t\left(x_{1}-x_{2}\right)\right)-\nabla f\left(x^{*}+\beta_{i}\left(x_{1}-x^{*}\right)\right)\right\| d t,
$$

which implies

$$
A \leq K_{1} \sum_{i=1}^{M}\left|a_{i}\right|\left\|x_{1}-x_{2}\right\| \int_{0}^{1}\left\|x_{2}+t\left(x_{1}-x_{2}\right)-x^{*}-\beta_{i}\left(x_{1}-x^{*}\right)\right\| d t .
$$

Since $x_{1}, x_{2} \in B_{\delta}\left(x^{*}\right)$, we find

$$
A \leq K_{1} \delta\left[\sum_{i=1}^{M}\left|a_{i}\right|\left(1+\left|\beta_{i}\right|\right)\right]\left\|x_{1}-x_{2}\right\| .
$$

In a similar way, one also obtains

$$
B \leq 2 K_{1} \delta\left[\sum_{i=1}^{M}\left|a_{i}\right|\left|\beta_{i}\right|\right]\left\|x_{1}-x_{2}\right\|
$$

By (7), (8), and (9), we deduce that

$$
\forall x_{1}, x_{2} \in B_{\delta}\left(x^{*}\right), \quad\left\|h\left(x_{1}\right)-h\left(x_{2}\right)\right\| \leq 2 K_{1} \delta\left[\sum_{i=1}^{M}\left|a_{i}\right|\left(1+\left|\beta_{i}\right|\right)\right]\left\|x_{1}-x_{2}\right\| .
$$

According to (10), and the fact that $\delta \leq \alpha_{1}$, we have

$$
\forall x_{1}, x_{2} \in B_{\delta}\left(x^{*}\right), \quad\left\|h\left(x_{1}\right)-h\left(x_{2}\right)\right\| \leq \epsilon\left\|x_{1}-x_{2}\right\|,
$$

which achieves the proof of the proposition.

Proposition 2.3. Under the assumptions of theorem 2.1, there exists $\delta>0$ such that, for all $x_{0} \in B_{\delta}\left(x^{*}\right)$ and $x_{0} \neq x^{*}$, the map $\Psi_{0}$ admits a fixed point $x_{1} \in B_{\delta}\left(x^{*}\right)$.

Proof. For the proof of this proposition, we show both assertions (i) and (ii) of lemma 1.3. 
The assumption $(\mathcal{H} 3)$ gives the constants $a$ and $b$; moreover we set

$$
\begin{aligned}
\alpha_{2} & =\sqrt{\frac{2 b}{3 K_{1}\left[\sum_{i=1}^{M}\left|a_{i}\right|\left(1+2\left|\beta_{i}\right|\right)\right]}} \\
\alpha_{3} & =\sqrt{\frac{1}{c}} \\
\alpha_{4} & =\sqrt[3]{\frac{6 b}{K_{2}\left[1+3 \sum_{i=1}^{M}\left|a_{i}\right| \beta_{i}^{2}\right]}}
\end{aligned}
$$

Let us consider $\delta>0$ such that

$$
\delta<\min \left\{a, \alpha_{2}, \alpha_{3}, \alpha_{4}\right\}
$$

From the definition of the excess $e$, we have

$$
\operatorname{dist}\left(x^{*}, \Psi_{0}\left(x^{*}\right)\right) \leq e\left(Q^{-1}(0) \cap B_{\delta}\left(x^{*}\right), Q^{-1}\left(\Lambda_{x^{*}}\left(x^{*}\right)-\Lambda_{0}\left(x^{*}\right)\right)\right) .
$$

We have

$$
\left\|\Lambda_{x^{*}}\left(x^{*}\right)-\Lambda_{0}\left(x^{*}\right)\right\|=\left\|f\left(x^{*}\right)-f\left(x_{0}\right)-\sum_{i=1}^{M} a_{i} \nabla f\left(x_{0}+\beta_{i}\left(x^{*}-x_{0}\right)\right)\left(x^{*}-x_{0}\right)\right\|,
$$

which can be rewritten

$$
\begin{aligned}
\left\|\Lambda_{x^{*}}\left(x^{*}\right)-\Lambda_{0}\left(x^{*}\right)\right\|= & \| f\left(x^{*}\right)-f\left(x_{0}\right)-\nabla f\left(x_{0}\right)\left(x^{*}-x_{0}\right)-\frac{1}{2} \nabla^{2} f\left(x_{0}\right)\left(x^{*}-x_{0}\right)^{2} \\
& +\nabla f\left(x_{0}\right)\left(x^{*}-x_{0}\right)+\frac{1}{2} \nabla^{2} f\left(x_{0}\right)\left(x^{*}-x_{0}\right)^{2} \\
& -\sum_{i=1}^{M} a_{i} \nabla f\left(x_{0}+\beta_{i}\left(x^{*}-x_{0}\right)\right)\left(x^{*}-x_{0}\right) \|
\end{aligned}
$$

Let us also set

and

$$
D=\left\|f\left(x^{*}\right)-f\left(x_{0}\right)-\nabla f\left(x_{0}\right)\left(x^{*}-x_{0}\right)-\frac{1}{2} \nabla^{2} f\left(x_{0}\right)\left(x^{*}-x_{0}\right)^{2}\right\|
$$

$$
\begin{aligned}
E= & \| \nabla f\left(x_{0}\right)\left(x^{*}-x_{0}\right)+\frac{1}{2} \nabla^{2} f\left(x_{0}\right)\left(x^{*}-x_{0}\right)^{2} \\
& -\sum_{i=1}^{M} a_{i} \nabla f\left(x_{0}+\beta_{i}\left(x^{*}-x_{0}\right)\right)\left(x^{*}-x_{0}\right) \| .
\end{aligned}
$$


Using $(\mathcal{H} 1)$, we obtain

$$
D \leq \frac{K_{2}}{6}\left\|x^{*}-x_{0}\right\|^{3}
$$

Since

$$
\nabla f\left(x_{0}+\beta_{i}\left(x^{*}-x_{0}\right)\right)-\nabla f\left(x_{0}\right)=\beta_{i} \int_{0}^{1} \nabla^{2} f\left(x_{0}+\beta_{i} t\left(x^{*}-x_{0}\right)\right)\left(x^{*}-x_{0}\right) d t
$$

we have

$$
E \leq \sum_{i=1}^{M}\left|a_{i} \beta_{i}\right|\left\|x^{*}-x_{0}\right\|^{2} \int_{0}^{1}\left\|\nabla^{2} f\left(x_{0}+\beta_{i} t\left(x^{*}-x_{0}\right)\right)-\nabla^{2} f\left(x_{0}\right)\right\| d t .
$$

The use of $(\mathcal{H} 1)$ gives also

$$
E \leq \frac{K_{2}}{2}\left[\sum_{i=1}^{M}\left|a_{i}\right| \beta_{i}^{2}\right]\left\|x^{*}-x_{0}\right\|^{3} .
$$

According to (11), (12), (13), (14), and using the pseudo-Lipschitzness of $Q^{-1}$, we obtain

$$
\left\|\Lambda_{x^{*}}\left(x^{*}\right)-\Lambda_{0}\left(x^{*}\right)\right\| \leq b
$$

and

$$
\operatorname{dist}\left(x^{*}, \Psi_{0}\left(x^{*}\right)\right) \leq L\left\|\Lambda_{x^{*}}\left(x^{*}\right)-\Lambda_{0}\left(x^{*}\right)\right\| .
$$

Using (12), (13), and (14) one has

$$
\operatorname{dist}\left(x^{*}, \Psi_{0}\left(x^{*}\right)\right) \leq \frac{L K_{2}}{6}\left(1+3 \sum_{i=1}^{M}\left|a_{i}\right| \beta_{i}^{2}\right)\left\|x^{*}-x_{0}\right\|^{3} .
$$

By setting

$$
r=r_{0}=c\left\|x^{*}-x_{0}\right\|^{3}, \quad \lambda=L K_{1} \delta\left[\sum_{i=1}^{M}\left|a_{i}\right|\left(1+4\left|\beta_{i}\right|\right)\right],
$$

since

$$
c>\frac{L K_{2}}{6}\left(1+3 \sum_{i=1}^{M}\left|a_{i}\right| \beta_{i}^{2}\right),
$$

one can decrease $\delta$ if it is necessary so that $\lambda \in] 0,1[$ and

$$
c(1-\lambda)>\frac{L K_{2}}{6}\left(1+3 \sum_{i=1}^{M}\left|a_{i}\right| \beta_{i}^{2}\right)
$$


and assertion (i) in lemma 1.3 is satisfied.

Let us remark that the decreasing of $\delta$ implies that the number $r_{0}=r_{0}(\delta)$ also decreases and the above choice of $r_{0}$ and (11) imply $r_{0} \leq \delta<a$.

Now, let us show that condition (ii) is also satisfied. Let $x \in B_{\delta}\left(x^{*}\right)$ and let us set $y=\Lambda_{x^{*}}(x)-\Lambda_{0}(x)$. We have

$$
\begin{aligned}
\|y\| \leq & \left\|f\left(x^{*}\right)-f\left(x_{0}\right)-\sum_{i=1}^{M} a_{i} \nabla f\left(x_{0}+\beta_{i}\left(x-x_{0}\right)\right)\left(x^{*}-x_{0}\right)\right\| \\
& +\|\left(\sum_{i=1}^{M} a_{i} \nabla f\left(x^{*}+\beta_{i}\left(x-x^{*}\right)\right)\right. \\
& \left.-\sum_{i=1}^{M} a_{i} \nabla f\left(x_{0}+\beta_{i}\left(x-x_{0}\right)\right)\right)\left(x-x^{*}\right) \| .
\end{aligned}
$$

Let us also set

$$
F=\left\|f\left(x^{*}\right)-f\left(x_{0}\right)-\sum_{i=1}^{M} a_{i} \nabla f\left(x_{0}+\beta_{i}\left(x-x_{0}\right)\right)\left(x^{*}-x_{0}\right)\right\|
$$

and

$$
G=\left\|\left(\sum_{i=1}^{M} a_{i} \nabla f\left(x^{*}+\beta_{i}\left(x-x^{*}\right)\right)-\sum_{i=1}^{M} a_{i} \nabla f\left(x_{0}+\beta_{i}\left(x-x_{0}\right)\right)\right)\left(x-x^{*}\right)\right\| .
$$

Using (3), we have

$$
\begin{aligned}
F & =\left\|\sum_{i=1}^{M} a_{i}\left(f\left(x^{*}\right)-f\left(x_{0}\right)-\nabla f\left(x_{0}+\beta_{i}\left(x-x_{0}\right)\right)\left(x^{*}-x_{0}\right)\right)\right\| \\
& =\left\|\sum_{i=1}^{M} a_{i} \int_{0}^{1}\left(\nabla f\left(x_{0}+t\left(x^{*}-x_{0}\right)\right)-\nabla f\left(x_{0}+\beta_{i}\left(x-x_{0}\right)\right)\right)\left(x^{*}-x_{0}\right) d t\right\| .
\end{aligned}
$$

Thus,

$$
F \leq \sum_{i=1}^{M}\left|a_{i}\right|\left\|x^{*}-x_{0}\right\| \int_{0}^{1}\left\|\nabla f\left(x_{0}+t\left(x^{*}-x_{0}\right)\right)-\nabla f\left(x_{0}+\beta_{i}\left(x-x_{0}\right)\right)\right\| d t .
$$

Since $x$ and $x_{0}$ belong to $B_{\delta}\left(x^{*}\right)$, we obtain

$$
F \leq \frac{K_{1} \delta^{2}}{2} \sum_{i=1}^{M}\left|a_{i}\right|\left(1+4\left|\beta_{i}\right|\right)
$$


In a similar way we obtain

$$
G \leq \delta^{2} K_{1} \sum_{i=1}^{M}\left|a_{i}\right|\left(1+\left|\beta_{i}\right|\right) .
$$

Thanks to (15), (16), and (17) one has

$$
\|y\| \leq \frac{3 K_{1} \delta^{2}}{2} \sum_{i=1}^{M}\left|a_{i}\right|\left(1+2\left|\beta_{i}\right|\right),
$$

and the inequality (11) implies $\|y\| \leq b$.

Let us set $H=e\left(\Psi_{0}(x) \cap B_{r_{0}}\left(x^{*}\right), \Psi_{0}\left(x^{\prime}\right)\right)$. It follows that, for all $x, x^{\prime} \in B_{r_{0}}\left(x^{*}\right)$, we have

$$
\begin{aligned}
H \leq & e\left(\Psi_{0}(x) \cap B_{\delta}\left(x^{*}\right), \Psi_{0}\left(x^{\prime}\right)\right) \\
\leq & L \| \sum_{i=1}^{M} a_{i} \nabla f\left(x^{*}+\beta_{i}\left(x-x^{*}\right)\right)\left(x-x^{*}\right)-\sum_{i=1}^{M} a_{i} \nabla f\left(x_{0}+\beta_{i}\left(x-x_{0}\right)\right)\left(x-x_{0}\right) \\
& -\sum_{i=1}^{M} a_{i} \nabla f\left(x^{*}+\beta_{i}\left(x^{\prime}-x^{*}\right)\right)\left(x^{\prime}-x^{*}\right)+\sum_{i=1}^{M} a_{i} \nabla f\left(x_{0}+\beta_{i}\left(x^{\prime}-x_{0}\right)\right)\left(x^{\prime}-x_{0}\right) \| .
\end{aligned}
$$

Thus,

$$
\begin{aligned}
H \leq & \| \sum_{i=1}^{M} a_{i} \nabla f\left(x^{*}+\beta_{i}\left(x-x^{*}\right)\right)\left(x-x^{\prime}\right)+\sum_{i=1}^{M} a_{i} \nabla f\left(x^{*}+\beta_{i}\left(x-x^{*}\right)\right)\left(x^{\prime}-x^{*}\right) \\
& -\sum_{i=1}^{M} a_{i} \nabla f\left(x^{*}+\beta_{i}\left(x^{\prime}-x^{*}\right)\right)\left(x^{\prime}-x^{*}\right)-\sum_{i=1}^{M} a_{i} \nabla f\left(x_{0}+\beta_{i}\left(x-x_{0}\right)\right)\left(x-x^{\prime}\right) \\
& -\sum_{i=1}^{M} a_{i} \nabla f\left(x_{0}+\beta_{i}\left(x-x_{0}\right)\right)\left(x^{\prime}-x_{0}\right)+\sum_{i=1}^{M} a_{i} \nabla f\left(x_{0}+\beta_{i}\left(x^{\prime}-x_{0}\right)\right)\left(x^{\prime}-x_{0}\right) \|,
\end{aligned}
$$

which yields

$$
\begin{aligned}
H \leq & L\left\|\left(\sum_{i=1}^{M} a_{i} \nabla f\left(x^{*}+\beta_{i}\left(x-x^{*}\right)\right)-\sum_{i=1}^{M} a_{i} \nabla f\left(x_{0}+\beta_{i}\left(x-x_{0}\right)\right)\right)\left(x-x^{\prime}\right)\right\| \\
& +L\left\|\left(\sum_{i=1}^{M} a_{i} \nabla f\left(x^{*}+\beta_{i}\left(x-x^{*}\right)\right)-\sum_{i=1}^{M} a_{i} \nabla f\left(x^{*}+\beta_{i}\left(x^{\prime}-x^{*}\right)\right)\right)\left(x^{\prime}-x^{*}\right)\right\| \\
& +\left\|\left(\sum_{i=1}^{M} a_{i} \nabla f\left(x_{0}+\beta_{i}\left(x^{\prime}-x_{0}\right)\right)-\sum_{i=1}^{M} a_{i} \nabla f\left(x_{0}+\beta_{i}\left(x-x_{0}\right)\right)\right)\left(x^{\prime}-x_{0}\right)\right\| .
\end{aligned}
$$


The last inequality implies

$$
H \leq L K_{1} \delta\left[\sum_{i=1}^{M}\left|a_{i}\right|\left(1+4\left|\beta_{i}\right|\right)\right]\left\|x-x^{\prime}\right\|,
$$

then

$$
e\left(\Psi_{0}(x) \cap B_{r_{0}}\left(x^{*}\right), \Psi_{0}\left(x^{\prime}\right)\right) \leq L K_{1} \delta\left[\sum_{i=1}^{M}\left|a_{i}\right|\left(1+4\left|\beta_{i}\right|\right)\right]\left\|x-x^{\prime}\right\|,
$$

and using (11), the condition (ii) in lemma 1.3 is satisfied.

Applying this lemma we get the existence of a fixed point $x_{1} \in B_{r_{0}}\left(x^{*}\right)$ of $\Psi_{0}$.

Moreover, $x_{1}$ in the preceding proposition satisfies the inequality (6). Proceeding by induction, assuming that $x_{k} \in B_{r_{k-1}}\left(x^{*}\right)$, keeping $\eta_{k}=x^{*}$, and setting $r_{k}=c\left\|x_{k}-x^{*}\right\|^{3}$, we obtain the existence of a fixed point $x_{k+1} \in B_{r_{k}}\left(x^{*}\right)$ for $\Psi_{k}$. This implies

$$
\left\|x_{k+1}-x^{*}\right\| \leq c\left\|x_{k}-x^{*}\right\|^{3} .
$$

In other words, $\left(x_{k}\right)_{k \geq 0}$ is cubically convergent to $x^{*}$, which completes the proof of theorem 2.1.

Concluding remarks.

- If $M=1, a_{1}=1$, and $\beta_{1}=0,(2)$ is the Newton-type sequence for solving (1).

- If $M=2, a_{1}=a_{2}=\frac{1}{2}, \beta_{1}=0$, and $\beta_{2}=1,(2)$ is the sequence studied in [12].

Acknowledgement. The authors thank the anonymous referee for his valuable remarks and comments, which improved the presentation of this manuscript.

\section{References}

[1] J.-P. Aubin, Lipschitz behavior of solutions to convex minimization problems, Math. Oper. Res. 9 (1984), no. 1, 87-111.

[2] J.-P. Aubin and H. Frankowska, Set-valued analysis, Systems \& Control: Foundations \& Applications, vol. 2, Birkhäuser Boston Inc., Boston, MA, 1990.

[3] A. L. Dontchev, Local convergence of the Newton method for generalized equations, C. R. Acad. Sci. Paris Sér. I Math. 322 (1996), no. 4, 327-331.

[4] Uniform convergence of the Newton method for Aubin continuous maps, Serdica Math. J. 22 (1996), no. 3, 283-296. Well-posedness and stability of variational problems.

[5] A. L. Dontchev and W. W. Hager, An inverse mapping theorem for set-valued maps, Proc. Amer. Math. Soc. 121 (1994), no. 2, 481-489.

[6] A. L. Dontchev, M. Quincampoix, and N. Zlateva, Aubin criterion for metric regularity, J. Convex Anal. 13 (2006), no. 2, 281-297. 
[7] M. H. Geoffroy, S. Hilout, and A. Piétrus, Acceleration of convergence in Dontchev's iterative method for solving variational inclusions, Serdica Math. J. 29 (2003), no. 1, 45-54.

[8] Stability of a cubically convergent method for generalized equations, Set-Valued Anal. 14 (2006), no. 1, 41-54.

[9] M. H. Geoffroy, C. Jean-Alexis, and A. Piétrus, A Hummel-Seebeck type method for solving variational inclusions, preprint.

[10] M. H. Geoffroy and A. Piétrus, A superquadratic method for solving generalized equations in the Hölder case, Ricerche Mat. 52 (2003), no. 2, 231-240 (2004).

[11] A. D. Ioffe and V. M. Tihomirov, Theory of extremal problems, Studies in Mathematics and its Applications, vol. 6, North-Holland Publishing Co., Amsterdam, 1979.

[12] C. Jean-Alexis, A cubic method without second-order derivative for solving variational inclusions, C. R. Acad. Bulgare Sci. 59 (2006), no. 12, 1213-1218.

[13] B. Mordukhovich, Complete characterization of openness, metric regularity, and Lipschitzian properties of multifunctions, Trans. Amer. Math. Soc. 340 (1993), no. 1, 1-35.

[14] _ Stability theory for parametric generalized equations and variational inequalities via nonsmooth analysis, Trans. Amer. Math. Soc. 343 (1994), no. 2, 609-657.

[15] A. Piétrus, Does Newton's method for set-valued maps converges uniformly in mild differentiability context?, Rev. Colombiana Mat. 34 (2000), no. 2, 49-56.

[16] _ Generalized equations under mild differentiability conditions, Rev. R. Acad. Cienc. Exactas Fís. Nat. (Esp.) 94 (2000), no. 1, 15-18.

[17] R. T. Rockafellar, Lipschitzian properties of multifunctions, Nonlinear Anal. 9 (1985), no. 8, $867-885$.

[18] R. T. Rockafellar and R. J.-B. Wets, Variational analysis, Grundlehren der Mathematischen Wissenschaften, vol. 317, Springer-Verlag, Berlin, 1998.

[19] J. F. Traub, Iterative methods for the solution of equations, Chelsea Publishing company, NewYork, 1985. 